\title{
TRACE CLASS SELF-COMMUTATORS
}

BY

\author{
C. A. BERGER AND MARION GLAZERMAN BEN-JACOB
}

\begin{abstract}
This paper extends earlier results of Berger and Shaw to all $W^{*}$ algebras. The multiplicity of an operator in a $W^{*}$ algebra is defined in terms of the trace on the $W^{*}$-algebra, and it is shown that if $T$ is a hyponormal operator in such an algebra, the trace of its self-commutator is bounded by this multiplicity times the area of the spectrum of $T$, divided by $\pi$.
\end{abstract}

Let $A$ be an operator on a Hilbert space $H$. The self-commutator of $A,\left[A^{*}, A\right]$, is defined by $\left[A^{*}, A\right]=A^{*} A-A A^{*}$. Earlier results provided bounds on the trace of a self-commutator valid for operators in the $I_{\infty}$ von Neumann factor $B(H)$. Let $G$ be a set of vectors in $H$ and let $|G|$ denote the cardinality of $G$. Let $T$ be a hyponormal operator in $B(H)$ such that the rational functions in $T$ acting on $G$ form a dense subspace of $H$. Berger and Shaw showed that $\operatorname{tr}\left[T^{*}, T\right] \leqslant \pi^{-1}|G|$ - area(spectrum $T$ ) [2]. The authors prove a version of this result meaningful in an arbitrary von Neumann algebra context.

Each section in this paper deals with a theorem that is an essential tool for the proof of the major result in $\S 4$.

Preliminaries. $Q_{H}$ will denote a von Neumann algebra of operators on the Hilbert space $H$. All Hilbert spaces are complex and tr and $\tau$ are used to denote normal traces. When the symbols for the trace function are subscripted, the subscript refers to the algebra of operators upon which the function is acting. The same capital letter will represent both a space and the projection onto the space. The set of rational functions with poles off a compact set $F$ will be denoted by $Q(F)$. If $\mu$ is a finite regular measure with compact support $E, Q^{2}(F, \mu)$ is the closure of rational functions with poles off $F$ in $L^{2}(\mu)$ for any compact set $F$ in $E$. Rational functions in an operator $T$ will be denoted by $q(T)$ and polynomials in $T$ by $p(T)$. $P$ will represent the set of analytic polynomials and $P^{\prime}$ will be $P \backslash\{0\}$. Planar Lebesgue measure will be denoted by $\eta$. Other notations are standard; e.g., $B(H, K)$ is the set of bounded operators from $H$ to $K$, and $B(H)=B(H, H)$.

The following definitions are fundamental. Let $T$ be an operator and $V$ a projection in $\mathcal{Q}_{H}$. Then $\langle v\rangle_{T}$ is the closure of the set $\left\{\sum_{i=1}^{m} p_{i}(T) v_{i}: p_{i} \in P, v_{i} \in V\right\}$ and $\langle v\rangle_{T}^{\prime}$ is the closure of the set $\left\{\sum_{i=1}^{m} q_{i}(T) v_{i}: q_{i} \in Q(\operatorname{sp}(T)), v_{i} \in V\right\}$.

Received by the editors September 11, 1981.

1980 Mathematics Subject Classification. Primary 47B20; Secondary 47C15, 47B10, $47 \mathrm{~B} 47$.

Key words and phrases. Normal trace, $W^{*}$-algebra, von Neumann algebra, hyponormal operator, self-commutator. 
(i) If $\langle v\rangle_{T}=H$, then $V$ is said to be polynomially cyclic for $T$. The polynomial multiplicity of $T$ is the infimum of all values $\operatorname{tr}(V)$ such that $V$ is in $Q_{H}$ and $V$ is polynomially cyclic for $T$.

(ii) If $\langle v\rangle_{T}^{\prime}=H, V$ is said to be rationally cyclic for $T$. The infimum of all values $\operatorname{tr}(V)$ such that $V$ is in $Q_{H}$ and $V$ is rationally cyclic for $T$ is the rational multiplicity of $T$.

(iii) If $\left\{x: \exists p \in P^{\prime}\right.$ such that $\left.p(T) x \in\langle v\rangle_{T}\right\}$ is dense in $H$, then $V$ is said to be effectually rationally cyclic for $T$. The effectual rational multiplicity of $T$ is the infimum of all values $\operatorname{tr}(V)$ such that $V$ is effectually rationally cyclic for $T$.

The major result we present is the following theorem.

THEOREM 4.1. Let $A$ be a hyponormal operator in $\mathcal{Q}_{H}$ with effectual rational multiplicity $s<+\infty$. Then

$$
\operatorname{tr}\left[A^{*}, A\right] \leqslant(s / \pi) \operatorname{area}(\operatorname{sp}(T)) .
$$

1. Let $\mathcal{Q}_{H}, \mathcal{Q}_{K}$ be von Neumann algebras in $B(H), B(K)$, respectively. Let $W \in B(H, K)$ such that $W^{*} \mathbb{Q}_{K} W \subseteq \mathbb{Q}_{H}, W \mathbb{Q}_{H} W^{*} \subseteq \mathbb{Q}_{K}$.

Let $\mathbb{Q}_{H, K}(W)$ be the weak closure of the set $\left\{B W A: B \in \mathbb{Q}_{K}, A \in \mathbb{Q}_{H}\right\}$. It follows that $\mathcal{Q}_{K, \underline{H}}\left(W^{*}\right)=\mathbb{Q}_{H, K}(W)^{*}$. Writing $W=V \sqrt{W^{*} W}$, where $V$ is the partial isometry from $R\left(W^{*}\right)$ to $R(W), Q_{H, K}(W)=Q_{K} V Q_{H}=V Q_{H}$.

Let $\mathcal{Q}$ be the von Neumann algebra in $B(H, K)$ consisting of operators

$$
\left(\begin{array}{ll}
A & Y \\
Y^{\prime} & B
\end{array}\right)
$$

such that $A \in \mathbb{Q}_{H}, B \in \mathbb{Q}_{K}, Y^{\prime} \in \mathbb{Q}_{H, K}(W)$ and $Y \in \mathbb{Q}_{K, H}\left(W^{*}\right)$.

We identify $Q_{H}$ with the embedded algebra $\left(\begin{array}{cc}A & 0 \\ 0 & 0\end{array}\right)$ in $\mathcal{W} S$ and $Q_{K}$ with the embedded algebra $\left(\begin{array}{ll}0 & 0 \\ 0 & B\end{array}\right)$ in $Q$.

LEMMA 1.1. Let $Q$ be a von Neumann algebra with a real valued trace $\tau$. Let $X$ be a positive hermitian operator and $P$ a projection in $Q$. Then $X$ lies in trace class if and only if $(I-P) X(I-P)$ and $P X P$ do.

Proof. The operator

$$
\begin{aligned}
X & =[P+(I-P)] X[P+(I-P)] \\
& =P X P+(I-P) X(I-P)+(I-P) X P+P X(I-P) .
\end{aligned}
$$

Trace class operators form an ideal, therefore if $X$ is in trace class so are $P X P$ and $(I-P) X(I-P), P X(I-P)$ and $(I-P) X P$.

Conversely, suppose

$$
X=\left(\begin{array}{ll}
X_{11} & X_{12} \\
X_{21} & X_{22}
\end{array}\right)
$$

is a positive hermitian operator and $X_{11}$ and $X_{22}$ are in trace class. We can write $X=Y^{*} Y$, where $Y$ is in $W$ and

$$
X_{11}=Y_{11}^{*} Y_{11}+Y_{12}^{*} Y_{21}, \quad X_{22}=Y_{21}^{*} Y_{21}+Y_{22}^{*} Y_{22} \text {. }
$$

Thus, $Y_{11}^{*} Y_{11}, Y_{12}^{*} Y_{12}, Y_{21}^{*} Y_{21}$ and $Y_{22}^{*} Y_{22}$ are individually in trace class. Thus, each $Y_{i j}$ is a Hilbert-Schmidt operator, so $Y$ is. Thus, $X=Y^{*} Y$ is in trace class. 
In the following three lemmas, $W$ is an operator in $B(H, K)$, and $W=V \sqrt{W^{*} W}$ is its polar decomposition. Both $\mathcal{Q}_{H}$ and $\mathcal{Q}_{K}$ are von Neumann algebras, and $W^{*} \mathbb{Q}_{K} W \subset \mathbb{Q}_{H}, W \mathbb{Q}_{H} W^{*} \subset \mathbb{Q}_{K}$. Note that then $V^{*} \mathbb{Q}_{K} V \subset \mathbb{Q}_{H}, V \mathbb{Q}_{H} V^{*} \subset \mathbb{Q}_{K}$.

LEMMA 1.2. If $W$ has dense range and $\tau_{\mathfrak{Q}_{H}}$ is a normal trace on $\mathbb{Q}_{H}$, then there is a normal trace $\tau_{\mathbb{Q}_{K}}$ on $\mathbb{Q}_{K}$ such that if $P_{H}$ is a projection in $\mathbb{Q}_{H}$ contained in $\overline{R\left(W^{*}\right)}$, then $\tau_{\mathbb{Q}_{K}}\left(V P_{H} V^{*}\right)=\tau_{\mathbb{Q}_{H}}\left(P_{H}\right)$. In particular, if $E(\sigma)$ is the spectral projection for $W^{*} W$ and $F(\sigma)$ is the spectral projection for $W W^{*}$, then

$$
\tau_{\mathbb{Q}_{H}}(E(\sigma))=\tau_{\mathbb{Q}_{K}}(F(\sigma)) .
$$

Proof. Since $R(V)=\overline{R(W)}=K, V^{*} \mathbb{Q}_{K} V$ in $\mathbb{Q}_{H}$ and $V \mathbb{Q}_{H} V^{*}$ in $\mathbb{Q}_{K}$ together imply $\mathbb{Q}_{K}=V \mathbb{Q}_{H} V^{*}$. It suffices to define $\tau_{\mathbb{Q}_{K}}$ on projections in $\mathbb{Q}_{K}$. The operator $V$ is an isometry from $\overline{R\left(W^{*}\right)}=\overline{R \sqrt{W^{*} W}}$ onto $K$. Given a projection $P_{K}$ in $Q_{K}$, there exists a unique projection $P_{H}$ in $Q_{H}$, contained in $\overline{R \sqrt{W^{*} W}}$, such that $P_{K}=V P_{H} V^{*}$. If we define $\tau_{\mathfrak{Q}_{K}}\left(P_{K}\right)=\tau_{\mathfrak{Q}_{K}}\left(V P_{H} V^{*}\right)=\tau_{\mathfrak{Q}_{H}}\left(P_{H}\right), \tau_{\mathfrak{Q}_{K}}$ is well defined. Moreover, $F(\sigma)$ $=V E(\sigma) V^{*}$ and the result follows.

LEMMA 1.3.1. Let

$$
\mathbb{Q}_{H} \otimes M_{2}=\left(\begin{array}{ll}
\mathbb{Q}_{H} & \mathbb{Q}_{H} \\
\mathbb{Q}_{H} & \mathbb{Q}_{H}
\end{array}\right) .
$$

Let $\tilde{e}$ be the embedding map of $Q_{H}$ into $Q_{H} \otimes M_{2}$. That is,

$$
\tilde{A}=\tilde{e}(A)=\left(\begin{array}{ll}
A & 0 \\
A & 0
\end{array}\right) \text { for } A \text { in } \mathbb{Q}_{H} \text {. }
$$

Let $Q$ be a projection in $Q_{H} \otimes M_{2}$. Then $Q=Q_{1}+Q_{2}$, where $Q_{1}$ and $Q_{2}$ are orthogonal projections such that $Q_{1}=V_{1}^{*} V_{1}$ and $Q_{2}=V_{2}^{*} V_{2}$, where $V_{1} V_{1}^{*}$ and $V_{2} V_{2}^{*}$ are contained in $\tilde{e}\left(\mathbb{Q}_{H}\right)$.

Proof. Let $Q$ be a projection in $Q_{H} \otimes M_{2}$ and let $P=\left(\begin{array}{ll}I & 0 \\ 0 & 0\end{array}\right)$. The operator $Q=Q P+Q(I-P)$. Let $Q P=W_{1} M_{1}$ and $Q(I-P)=W_{2} M_{2}$ be the polar decompositions for $Q P$ and $Q(I-P)$, respectively. The operator $M=\sqrt{P Q P}, R\left(M_{1}\right)$ is contained in $R(P), W_{1}^{*} W_{1}$ is contained in $P$, and $W_{1} W_{1}^{*}$ is in $Q$. Since $Q$ and $P$ belong to $Q_{H} \otimes M_{2}$, so do $W_{i}$ and $M_{i}$ for $i=1,2 . W_{1} W_{1}^{*}=\overline{R(Q P Q)}$ implies that $Q-W_{1} W_{1}^{*}=Q-R(Q P Q)$.

We claim $Q-W_{1} W_{1}^{*}$ is contained in $(I-P)$. It suffices to show $\left(Q-W_{1} W_{1}^{*}\right) P$ $=0$. We have $Q P=W_{1} M_{1}$ and $R\left(W_{1} W_{1}^{*}\right)=P\left(W_{1}\right)=\overline{R(Q P)}=\overline{R(Q P Q)}$. If $x$ is in $R\left(Q-W_{1} W_{1}^{*}\right)$, then $x$ is perpendicular to $R\left(W_{1} W_{1}^{*}\right)$ and $x$ is in $R(Q)$. Thus, $Q P Q=0$. Since $0=Q P Q x=Q P P Q x=(P Q)^{*}(P Q) x$, we see $0=P Q x=P x$. We have $P\left(Q-W_{1} W_{1}^{*}\right)=0$ or, in other words, $\left(Q-W_{1} W_{1}^{*}\right)+W_{1} W_{1}^{*}$ is a projection orthogonal to $P$. Express $Q$ as $Q=\left(Q-W_{1} W_{1}^{*}\right)+W_{1} W_{1}^{*}$. Since $\left(Q-W_{1} W_{1}^{*}\right)$ is orthogonal to $P$ it is of the form $\left(\begin{array}{ll}0 & 0 \\ 0 & X\end{array}\right)$. It is equivalent to a matrix of the form $\left(\begin{array}{ll}Y & 0 \\ 0 & 0\end{array}\right)$ by the unitary transformation $\left(\begin{array}{cc}0 & I \\ I & 0\end{array}\right)$. The operator $W_{1} W_{1}^{*}$ is equivalent to $W_{1}^{*} W_{1}$ which is contained in $P$. Let $Q_{1}=\left(Q-W_{1} W_{1}^{*}\right), Q_{2}=W_{1} W_{1}^{*}, V_{2}=W_{1}^{*}, V_{1} V_{1}^{*}=Y$ and the proof is complete.

LEMMA 1.3.2. Define $\tau_{\text {ข }}=\tau_{\mathfrak{Q}_{H}}+\tau_{\mathfrak{Q}_{K}}$. If $W \in B(H, K)$ has dense range, then $\tau_{\text {ข }}$ is a well-defined trace on W. If $\tau_{\mathfrak{Q}_{H}}$ and $\tau_{\mathfrak{Q}_{K}}$ are normal, so is $\tau_{\text {w. }}$. 
Proof. Once it is known that $\tau_{\text {ข }}$ is well defined, the final remark is trivial. Let $W^{*}=V^{*} \sqrt{W W^{*}}$. We note that $R\left(V^{*}\right)=R\left(W^{*}\right)$ and $R(V)=R(W)$. The operator $W$ is dense so $\overline{R(V)}=\overline{R(W)}$ and $V V^{*}=I_{K}$. Since $W Q_{H} W^{*}$ is in $\mathcal{Q}_{K}$ and $W^{*} Q_{K} W$ is in $\mathcal{Q}_{H}$, we find $V \mathbb{Q}_{H} V^{*}$ is contained in $\mathbb{Q}_{K}$ and $V^{*} \mathbb{Q}_{K} V$ is in $\mathcal{Q}_{H}$. Let $B$ be in $\mathbb{Q}_{K}$. $V^{*} B V$ is in $\mathbb{Q}_{H}$ implying $V\left(V^{*} B V\right) V^{*}=B$, and thus $V \mathbb{Q}_{H} V^{*}=\mathbb{Q}_{K}$. Using this fact and that $R\left(V^{*}\right)=R\left(W^{*}\right)$ and $R(V)=R(W)$, we find

$$
\mathscr{W}=\left(\begin{array}{cc}
\mathbb{Q}_{H} & \mathbb{Q}_{H} V^{*} \\
V \mathcal{Q}_{H} & V \mathbb{Q}_{H} V^{*}
\end{array}\right)=\left(\begin{array}{cc}
I & 0 \\
0 & V
\end{array}\right)\left(\begin{array}{cc}
\mathcal{Q}_{H} & \mathcal{Q}_{H} \\
\mathcal{Q}_{H} & \mathcal{Q}_{H}
\end{array}\right)\left(\begin{array}{cc}
I & 0 \\
0 & V^{*}
\end{array}\right) .
$$

Since

$$
\left(\begin{array}{cc}
I & 0 \\
0 & V^{*}
\end{array}\right)
$$

is an isometry, it suffices to show $\tau_{\mathfrak{Q}_{H}} \oplus \tau_{\mathbb{Q}_{H}}$ is well defined on the projections in $Q_{H} \otimes M_{2}$. Clearly $\tau_{\mathfrak{Q}_{H}} \oplus \tau_{\mathfrak{Q}_{H}}$ is linear, and thus we need only prove that if $\tilde{Q}$ and $R$ are two projections in $\mathcal{Q}_{H} \otimes M_{2}$ and $X^{*} X=\tilde{Q}$ and $X X^{*}=R$, then $\left(\tau_{\mathbb{Q}_{H}} \oplus \tau_{\mathbb{Q}_{H}}\right)(\tilde{Q})$ $=\left(\tau_{\mathscr{Q}_{H}} \oplus \tau_{\mathfrak{Q}_{H}}\right)(R)$. If $X^{*} X=\tilde{Q}$ and $X X^{*}=R, R\left(X^{*}\right)=R(\tilde{Q})$ and thus $X \tilde{Q} X^{*}=R$. From Lemma 1.3.1 we have $\tilde{Q}=Q_{1}+Q_{2}$, where the $Q_{i}$ are orthogonal projections, each equivalent to an embedded projection. We have $X \tilde{Q} X^{*}=X Q_{1} X^{*}+X Q_{2} X^{*}=$ $R_{1}+R_{2}=R$. Thus it suffices to show that $\operatorname{tr}\left(Q_{i}\right)=\operatorname{tr}\left(X Q_{i} X^{*}\right)=\operatorname{tr}\left(R_{i}\right)$ for $i=1,2$. Since $Q_{1}$ and $Q_{2}$ are equivalent to an embedded projection, we may, without any loss of generality, assume our original projection $\tilde{Q}$ was embedded; i.e. $\tilde{Q}=e(Q)$ for $Q$ in $\mathbb{Q}_{H}$. Let $X^{*} X=\tilde{Q}$ and $X X^{*}=R$. Since $X \tilde{Q} X^{*}=R$ and $R\left(X^{*}\right)=R(\tilde{Q})$, we have that

$$
X^{*}=\left(\begin{array}{ll}
X_{11}^{*} & X_{21}^{*} \\
0 & 0
\end{array}\right) \text { and } X=\left(\begin{array}{ll}
X_{11} & 0 \\
X_{21} & 0
\end{array}\right) .
$$

Thus, $Q=X_{11}^{*} X_{11}+X_{21}^{*} X_{21}$ and

$$
R=\left(\begin{array}{ll}
X_{11} & 0 \\
X_{21} & 0
\end{array}\right)\left(\begin{array}{ll}
X_{11}^{*} & X_{21}^{*} \\
0 & 0
\end{array}\right)=\left(\begin{array}{l}
X_{11} X_{11}^{*} \\
X_{21} V_{11}^{*}
\end{array}\right)\left(\begin{array}{l}
X_{11} X_{21}^{*} \\
X_{21} X_{21}^{*}
\end{array}\right) .
$$

To show $\operatorname{tr}(\tilde{Q})=\operatorname{tr}(R)$ we must show

$$
\operatorname{tr} \mathbb{Q}_{H}\left(X_{11}^{*} X_{11}\right)+\operatorname{tr} \mathbb{Q}_{H}\left(X_{21}^{*} X_{21}\right)=\operatorname{tr} \mathbb{Q}_{H}\left(X_{11} X_{11}^{*}\right)+\operatorname{tr} \mathbb{Q}_{H}\left(X_{21} X_{21}^{*}\right) .
$$

Since $\operatorname{tr} \mathbb{Q}_{H}$ is well defined on $\mathbb{Q}_{H}$ and $X_{i j}$ and $V_{i j}^{*}$ are in $\mathbb{Q}_{H}$ for $i, j=1,2$ we have that (1) is true.

The Transfer Theorem. Let $T$ in $\mathbb{Q}_{H}$ and $A$ in $\mathbb{Q}_{K}$ be hyponormal operators. Let $W$ belong to $B(H, K)$ and satisfy the following:

(i) $W^{*} \mathbb{Q}_{K} W \subset \mathbb{Q}_{H}, W \mathbb{Q}_{K} W^{*} \subset \mathbb{Q}_{H}$.

(ii) $W^{*} W$ and $W W^{*}$ are trace class operators in $\mathbb{Q}_{H}$ and $\mathbb{Q}_{K}$, respectively.

(iii) $\tau_{\mathfrak{Q}_{K}}\left(V X V^{*}\right)=\tau_{\mathfrak{Q}_{H}}(X)$ for all $X$ in trace class in $\mathbb{Q}_{H}$.

(iv) $W$ has dense range.

(v) $W T=A W$.

Then $\tau_{\mathbb{Q}_{H}}\left[A^{*}, A\right] \leqslant \tau_{\mathbb{Q}_{H}}\left[T^{*}, T\right]$. 
Proof. Let $J_{t}$ be the subspace of $H \oplus K$ defined by $J_{t}=\{t h \oplus W h \mid h \in H\} . J_{t}$ is invariant for $T \oplus A$ and therefore $\left.(T \oplus A)\right|_{J_{t}}$ is a hyponormal operator. After proving

$$
\operatorname{tr}\left[\left((T \oplus A) \mid J_{t}\right)^{*},\left.(T \oplus A)\right|_{J_{t}}\right]=\operatorname{tr}\left[T^{*}, T\right] \quad \text { when } t \rightarrow 0
$$

we will estimate $\operatorname{tr}\left[\left.(T \oplus A)\right|_{J_{t}} ^{*},\left.(T \oplus A)\right|_{J_{t}}\right]$.

Let $थ$ be as in Lemma 1.3.2 and let $\operatorname{tr}$ and $\tau$ be $\tau_{\text {ขs }}$. Let $Q$ be the map in $\mathscr{W}$ defined by $Q(h \oplus k)=t h \oplus W h$. Then

$$
Q T=\left.(T \oplus A)\right|_{J_{t}} Q \text { and }\left(Q^{*} Q\right)^{1 / 2}=t\left(I+W^{*} W / t^{2}\right)^{1 / 2}=t(I+M),
$$

where $M$ is a positive hermitian operator with finite trace. Thus, $Q=V_{J} t(I+M)$ and $\left.(T \oplus A)\right|_{J_{t}} V_{J}(I+M)=V_{J}(I+M) T$ leads us to

$$
\begin{aligned}
\operatorname{tr}\left[\left.(T \oplus A)\right|_{J_{t}} ^{*},\left.(T \oplus A)\right|_{J_{t}}\right] & =\operatorname{tr}\left[\left(\left.V_{J}^{*}(T \oplus A)\right|_{J_{t}} V_{J}\right)^{*},\left(\left.V_{J}^{*}(T \oplus A)\right|_{J_{t}} V_{J}\right)\right] \\
& =\operatorname{tr}\left[T^{*}, T\right] .
\end{aligned}
$$

Let $\{E(\sigma)\}$ and $\{F(\sigma)\}$ be the spectral resolutions for $W^{*} W$ and $W W^{*}$, respectively. If $Q=V_{J_{t}} M_{J_{t}}$ is the polar decomposition for $Q$, then $R(E(\sigma))$ is contained in $V_{J_{t}}^{*}$ and $E(\sigma) V_{J_{t}}^{*}$ is a partial isometry. Also, $(E(\sigma) \oplus 0)$ and $J_{t}(E(\sigma) \oplus F(\sigma)) J_{t}$ are equivalent projections and

$$
\tau_{\mathcal{Q}_{H}}(E(\sigma))=\tau_{\text {ชิ }}(E(\sigma) \oplus 0)=\tau\left(J_{t}(E(\sigma) \oplus F(\sigma)) J_{t}\right)=\tau\left[(E(\sigma) \oplus F(\sigma)) J_{t}\right] .
$$

We compute $J_{t}$ explicitly.

$$
\begin{aligned}
& J_{t}=\overline{R(Q)}=\overline{R\left(\begin{array}{cc}
t & 0 \\
W & 0
\end{array}\right)}, \quad Q=V \sqrt{Q^{*} Q} . \\
& Q^{*} Q=\left(\begin{array}{cc}
t^{2} I+W^{*} W & 0 \\
0 & 0
\end{array}\right), \quad \sqrt{\left(Q^{*} Q\right)}=\left(\begin{array}{cc}
\left(t^{2} I+W^{*} W\right)^{1 / 2} & 0 \\
0 & 0
\end{array}\right), \\
& V=Q\left(\sqrt{Q^{*} Q}\right)^{-1}=\left(\begin{array}{cc}
t & 0 \\
W & 0
\end{array}\right)\left(\begin{array}{cc}
\left(t^{2} I+W^{*} W\right)^{-1 / 2} & 0 \\
0 & 0
\end{array}\right) \\
& =\left(\begin{array}{cc}
t\left(t^{2} I+W^{*} W\right)^{-1 / 2} & 0 \\
W\left(t^{2} I+W^{*} W\right)^{-1 / 2} & 0
\end{array}\right) \\
& J_{t}=V V^{*} \\
& =\left(\begin{array}{cc}
t\left(t^{2} I+W^{*} W\right)^{-1 / 2} & 0 \\
W\left(t^{2} I+W^{*} W\right)^{-1 / 2} & 0
\end{array}\right)\left(\begin{array}{cc}
t\left(t^{2} I+W^{*} W\right)^{-1 / 2} & \left(t^{2} I+W^{*} W\right)^{-1 / 2} W^{*} \\
0 & 0
\end{array}\right) \\
& =\left(\begin{array}{cc}
t^{2}\left(t^{2} I+W^{*} W\right)^{-1} & t\left(t^{2} I+W^{*} W\right)^{-1} W^{*} \\
t W\left(t^{2} I+W^{*} W\right)^{-1} & W\left(t^{2} I+W^{*} W\right)^{-1} W^{*}
\end{array}\right) .
\end{aligned}
$$

For $0 \in \sigma$, let

$$
\begin{aligned}
\gamma(\sigma) & =\operatorname{tr}\left\{J_{t}(E(\sigma) \oplus F(\sigma)) J_{t}\left[\left.T \oplus A\right|_{J_{t}} ^{*},\left.T \oplus A\right|_{J_{t}}\right] J_{t}(E(\sigma) \oplus F(\sigma)) J_{t}\right\}, \\
\gamma(\{0\}) & =\operatorname{tr}\left\{J_{t}(E(\{0\}) \oplus 0) J_{t}\left[\left.T \oplus A\right|_{J_{t}} ^{*}\right] J_{t}(E(\{0\}) \oplus 0) J_{t}\right\} .
\end{aligned}
$$


We will evaluate

$$
\int_{[0,\|W\|]} d \gamma=\gamma(\{0\})+\int_{(0,\|W\|]} d \gamma
$$

since $\left\{J_{t}\{E(\sigma) \oplus F(\sigma)\} J_{t}\right\}$ is a spectral resolution for $J_{t}$. From Lemma 1.1 we see it suffices to examine (2) in order to estimate $\operatorname{tr}\left[\left.(T \oplus A)\right|_{J_{t}} ^{*},\left.(T \oplus A)\right|_{J_{t}}\right]$.

First consider

$$
\begin{aligned}
(E(\sigma) \oplus F(\sigma)) & J_{t}\left(T^{*} T \oplus A^{*} A\right) J_{t}(E(\sigma) \oplus F(\sigma)) \\
= & (E(\sigma) \oplus F(\sigma)) J_{t}\left(T^{*} T \oplus 0\right) J_{t}(E(\sigma) \oplus F(\sigma)) \\
& +(E(\sigma) \oplus F(\sigma)) J_{t}\left(0 \oplus A^{*} A\right) J_{t}(E(\sigma) \oplus F(\sigma)) .
\end{aligned}
$$

We claim, as $\tau$ approaches 0 , the trace of this expression goes to

$$
\operatorname{tr}\left[\left(0 \oplus A^{*} A\right) F(\sigma)\right]=\operatorname{tr}_{\mathbb{Q}_{K}}\left(A^{*} A F(\sigma)\right) .
$$

If $\sigma$ is a Borel set, bounded away from 0 in $(0,\|W\|], E(\sigma)$ and $F(\sigma)$ are trace class projections. Utilizing the properties of the trace function and the fact that

$$
(E(\sigma) \oplus F(\sigma)) J_{t}=J_{t}(E(\sigma) \oplus F(\sigma)),
$$

we find

$$
\begin{aligned}
\operatorname{tr}(E(\sigma) \oplus F(\sigma)) J_{t}\left(T^{*} T \oplus 0\right) J_{t}(E(\sigma) \oplus F(\sigma))-\operatorname{tr}\left[J_{t}\left(T^{*} T E(\sigma) \oplus 0\right) J_{t}\right] \\
=\operatorname{tr}\left[J_{t}\left(T^{*} T E(\sigma) \oplus 0\right)\right]=\operatorname{tr}\left(\begin{array}{cc}
t^{2}\left(t^{2} I+W^{*} W\right)^{-1} T^{*} T E(\sigma) & 0 \\
t W\left(t^{2} I+W^{*} W\right)^{-1} T^{*} T E(\sigma) & 0
\end{array}\right) \\
=\tau_{\mathfrak{Q}_{H}}\left(t^{2}\left(t^{2} I+W^{*} W\right)^{-1} T^{*} T E(\sigma)\right)=\tau_{\mathfrak{Q}_{H}}\left(t^{2}\left(t^{2} I+W^{*} W\right)^{-1} E(\sigma) T^{*} T\right) .
\end{aligned}
$$

Since $T^{*} T$ is a bounded operator, it suffices to show $t^{2}\left(t^{2} I+W^{*} W\right)^{-1} E(\sigma)$ goes to 0 in trace norm. Since this operator is positive, it suffices to show

$$
\tau_{\mathbb{Q}_{H}}\left(t^{2}\left(t^{2} I+W^{*} W\right)^{-1} E(\sigma)\right) \rightarrow 0 .
$$

This will be done later. Note that

$$
\tau_{\mathbb{Q}_{H}}\left(t^{2}\left(t^{2} I+W^{*} W\right)^{-1} E(\sigma)\right)=\operatorname{tr}\left((E(\sigma) \oplus 0) J_{t}\right) .
$$

We further claim

$$
\begin{aligned}
& \operatorname{tr}\left[(E(\sigma) \oplus F(\sigma)) J_{t}\left(0 \oplus A^{*} A\right)-J_{t}(E(\sigma) \oplus F(\sigma))\right] \\
& \quad=\operatorname{tr}\left[J_{t}\left(0 \oplus A^{*} A\right)(E(\sigma) \oplus F(\sigma)) J_{t}\right]=\operatorname{tr}\left[J_{t}\left(0 \oplus A^{*} A\right)(E(\sigma) \oplus F(\sigma))\right]
\end{aligned}
$$

approaches $\operatorname{tr}\left[0 \oplus A^{*} A F(\sigma)\right]$. In other words we want to show

$$
\operatorname{tr}\left[\left(I-J_{t}\right)\left(0 \oplus A^{*} A F(\sigma)\right)\right]
$$

goes to 0 as $t$ goes to 0 .

$$
\begin{aligned}
\operatorname{tr}\left(\left(I-J_{t}\right)\left(0 \oplus A^{*} A F(\sigma)\right)\right) & =\operatorname{tr}\left(\begin{array}{cc}
0 & -t\left(t^{2} I+W^{*} W\right)^{-1} W^{*} A^{*} A F(\sigma) \\
0 & W\left(t^{2} I+W^{*} W\right)^{-1} W^{*} A^{*} A F(\sigma)
\end{array}\right) \\
& =\tau_{\mathbb{Q}_{K}}\left(W\left(t^{2} I+W^{*} W\right)^{-1} W^{*} A^{*} A F(\sigma)\right)
\end{aligned}
$$


Thus, since $A^{*} A$ is bounded, it suffices to show $F(\sigma) W\left(t^{2} I+W^{*} W\right)^{-1} W^{*} \rightarrow 0$ in trace norm. But

$$
W\left(t^{2} I+W^{*} W\right)^{-1} W^{*}=W W\left(t^{2} I+W W^{*}\right)^{-1},
$$

which commutes with $F(\sigma)$. Thus, $F(\sigma) W\left(t^{2} I+W^{*} W\right)^{-1} W^{*}$ is positive, and it suffices to show $\tau_{\mathbb{Q}_{K}}\left(F(\sigma) W W^{*}\left(t^{2} I+W W^{*}\right)^{-1}\right) \rightarrow 0$. By the above, we have

$$
\tau_{\mathbb{Q}_{K}}\left(F(\sigma) W W^{*}\left(t^{2} I+W W^{*}\right)^{-1}\right)=\operatorname{tr}\left[\left(I-J_{t}\right)(0 \oplus F(\sigma))\right] .
$$

Both $\operatorname{tr}\left[(E(\sigma) \oplus 0) J_{t}\right]$ and $\operatorname{tr}\left[(0 \oplus F(\sigma)) J_{t}-(0 \oplus F(\sigma))\right]$ approaching 0 is equivalent to $\operatorname{tr}\left[(E(\sigma) \oplus F(\sigma)) J_{t}-(0 \oplus F(\sigma))\right]$ approaching 0 ; however by Lemmas 1.2 and 1.3.2 the last expression is equal to $\operatorname{tr}_{\mathbb{Q}_{K}}(F(\sigma))-\operatorname{tr}_{\mathscr{w}}(0 \oplus F(\sigma))$, which is 0 . Thus, it suffices to show $\operatorname{tr}\left(J_{t}(E(\sigma) \oplus 0)\right)$ goes to 0 as $t$ does to support both claims. Recall

$$
J_{t}=\left(\begin{array}{cc}
t^{2}\left(t^{2} I+W^{*} W\right)^{-1} & t\left(t^{2} I+W^{*} W\right)^{-1} W^{*} \\
t W\left(t^{2} I+W^{*} W\right)^{-1} & W\left(t^{2} I+W^{*} W\right)^{-1} W^{*}
\end{array}\right) .
$$

Thus,

$$
J_{t}(E(\sigma) \oplus 0)=\left(\begin{array}{cc}
t^{2}\left(t^{2} I+W^{*} W\right)^{-1} E(\sigma) & 0 \\
t W\left(t^{2} I+W^{*} W\right)^{-1} E(\sigma) & 0
\end{array}\right)
$$

and

$$
\begin{aligned}
\operatorname{tr}_{\mho} J_{t}(E(\sigma) \oplus 0) & =\operatorname{tr}_{\mathscr{Q}_{H}}\left[t^{2}\left(t^{2} I+W^{*} W\right)^{-1} E(\sigma)\right] \\
& =\int_{0^{+}}^{\left\|W^{*} W\right\|} \frac{t^{2}}{t^{2}+\lambda^{2}} d \tau(E(\lambda) E(\sigma)) .
\end{aligned}
$$

Since $t^{2} /\left(t^{2}+\lambda^{2}\right) \leqslant 1$, by the Lebesgue dominated convergence theorem

$$
\lim _{t \rightarrow 0} \int_{0^{+}}^{\left\|W^{*} W\right\|} \frac{t^{2}}{t^{2}+\lambda^{2}} d \tau(E(\lambda) E(\sigma))=0 .
$$

Now, let us consider

$$
\operatorname{tr}\left[(E(\sigma) \oplus F(\sigma))(T \oplus A) J_{t}\left(T^{*} \oplus A^{*}\right)(E(\sigma) \oplus F(\sigma))\right] .
$$

Writing each operator in (3) in matrix form, we find (3) equal to $\operatorname{tr}_{\mathfrak{Q}_{H}}\left(E(\sigma) t^{2} T\left(t^{2} I+W^{*} W\right)^{-1} T^{*} E(\sigma)\right)+\operatorname{tr}_{\mathfrak{Q}_{K}}\left(F(\sigma) A W^{*} W\left(t^{2} I+W^{*} W\right)^{-1} A F(\sigma)\right)$.

This expression is equal to

$$
\int_{0^{+}}^{\left\|W^{*} W\right\|} \frac{t^{2}}{t^{2}+\lambda^{2}} d \operatorname{tr}_{\mathfrak{Q}_{H}}(T E(\lambda) T(E(\sigma)))+\int_{0^{+}}^{\left\|W^{*} W\right\|} \frac{t^{2}}{t^{2}+\lambda^{2}} d \tau_{\mathfrak{Q}_{K}}\left(A F(\lambda) A^{*} F(\sigma)\right) .
$$

Both $t^{2} /\left(t^{2}+\lambda^{2}\right)$ and $\lambda^{2} /\left(t^{2}+\lambda^{2}\right)$ are bounded by 1 . By applying the Lebesgue dominated convergence theorem we see that as $t \rightarrow 0^{+}$, (4) approaches

$$
\begin{aligned}
\int_{0^{+}}^{\left\|W^{*} W\right\|} d \tau_{\mathbb{Q}_{K}}\left(A F(\lambda) A^{*} F(\sigma)\right) & =\tau_{\mathbb{Q}_{K}}\left(A F(0,\|W\|] A^{*} F(\sigma)\right) \\
& =\tau_{\mathbb{Q}_{K}}\left(A A^{*} F(\sigma)\right)=\tau_{\mathbb{Q}_{K}}\left(A A^{*} F(\sigma)\right)=\tau_{\mathbb{Q}_{K}}\left[F(\sigma) A A^{*} F(\sigma)\right] .
\end{aligned}
$$


Next, consider

$$
(E(\{0\}) \oplus 0)\left[J_{t}\left(T^{*} T \oplus A^{*} A\right) J_{t}-(T \oplus A) J_{t}\left(T^{*} \oplus A^{*}\right)\right](E(\{0\}) \oplus 0) .
$$

Using the fact that $[t E(\{0\}) \oplus 0]$ is contained in $J_{t}$ we find the operator

$$
(E(\{0\}) \oplus 0)\left[J_{t}\left(T^{*} T \oplus A^{*} A\right) J_{t}-(T \oplus A) J_{t}\left(T^{*} \oplus A^{*}\right)\right](E(\{0\}) \oplus 0)
$$

in matrix form is

$$
\begin{gathered}
\left(\begin{array}{cc}
E(\{0\}) t^{2}\left(t^{2} I+W^{*} W\right)^{-1} T^{*} T E(\{0\}) & 0 \\
0 & 0
\end{array}\right) \\
\qquad-\left(\begin{array}{ccc}
E(\{0\}) t^{2} T\left(t^{2} I+W^{*} W\right)^{-1} T^{*} E(\{0\}) & 0 \\
0 & 0
\end{array}\right) .
\end{gathered}
$$

We have

$$
\begin{gathered}
\tau_{\text {ข[ }}\left[(E(\{0\}) \oplus 0) J_{t}\left(T^{*} T \oplus A^{*} A\right) J_{t}-(T \oplus A) J_{t}\left(T^{*} \oplus A^{*}\right)(E(\{0\}) \oplus 0)\right] \\
=\tau_{\mathbb{Q}_{H}}\left[E(\{0\}) t^{2}\left(t^{2} I+W^{*} W\right)^{-1} T^{*} T E(\{0\})\right. \\
\left.-E(\{0\}) T t^{2}\left(t^{2} I+W^{*} W\right)^{-1} T^{*} E(\{0\})\right] .
\end{gathered}
$$

Set

$$
\begin{aligned}
Y & =\left[E(\{0\}) t^{2}\left(t^{2} I+W^{*} W\right)^{-1} T^{*} T E(\{0\})-E(\{0\}) T t^{2}\left(t^{2} I+W^{*} W\right)^{-1} T^{*} E(\{0\})\right] \\
& =Y-E(\{0\}) T T^{*} E(\{0\})+E(\{0\}) T T^{*} E(\{0\}) \\
& =E(\{0\})\left[T^{*}, T\right] E(\{0\})+E(\{0\}) T\left(\int_{0^{+}}^{\left\|W^{*} W\right\|} 1-\frac{t^{2}}{t^{2}+\lambda^{2}} d E(\lambda)\right) T^{*} E(\{0\}) \\
& =E(\{0\})\left[T^{*}, T\right] E(\{0\})+E(\{0\}) T\left(\int_{0^{+}}^{\left\|W^{*} W\right\|} \frac{\lambda^{2}}{t^{2}+\lambda^{2}} d E(\lambda)\right) T^{*} E(\{0\}) .
\end{aligned}
$$

Thus

$$
\begin{aligned}
\operatorname{tr}_{\mathfrak{Q}_{H}}(Y)= & \operatorname{tr}_{\mathfrak{Q}_{H}}\left[E(\{0\})\left[T^{*}, T\right] E(\{0\})\right] \\
& +\int_{0^{+}}^{\left\|W^{*} W\right\|} \frac{\lambda^{2}}{t^{2}+\lambda^{2}} d \tau_{\mathfrak{Q}_{H}}\left(E(\{0\}) T E(\lambda) T^{*} E(\{0\})\right) .
\end{aligned}
$$

The latter quantity is finite since

$$
\begin{aligned}
& \operatorname{tr}\left[T^{*}, T\right]=\operatorname{tr}_{\text {}}\left[(I-E(\{0\}))\left[\left.(T \oplus A)\right|_{J_{t}} ^{*},\left.(T \oplus A)\right|_{J_{t}}\right](I-E(\{0\}))\right] \\
& +_{\alpha_{H}}\left[E(\{0\})\left[T^{*}, T\right] E(\{0\})\right] \\
& +\int_{0^{+}}^{\left\|W^{*} W\right\|} \frac{\lambda^{2}}{t^{2}+\lambda^{2}} d \tau_{\mathbb{Q}_{H}}\left(E(\{0\}) T E(\lambda) T^{*} E(\{0\})\right) .
\end{aligned}
$$

The left-hand side of (5) is positive and finite. The first two terms on the right-hand side of the equation are positive, thus the last quantity, which is also positive, must 
be finite as well. Since the integrand increases monotonically to 1 as $t \rightarrow 0^{+}$, we see

$$
\int_{0^{+}}^{\left\|W^{*} W\right\|} 1 d \tau_{\mathfrak{Q}_{H}}\left(E(\{0\}) T E(\lambda) T^{*} E(\{0\})\right)
$$

is finite. Thus $\tau_{\mathbb{Q}_{H}}\left(E(\{0\}) T E(\lambda) T^{*} E(\{0\})\right)$ is a finite measure.

Now

$$
\begin{aligned}
Y= & Y-E(\{0\}) T E\{0\} T^{*} E(\{0\})+E(\{0\}) T E(\{0\}) T^{*} E(\{0\}) \\
= & {\left[(T E(\{0\}))^{*}, T E\{0\}\right]+E(\{0\}) T E(\{0\}) T^{*} E(\{0\}) } \\
& -E(\{0\}) T t^{2}\left(t^{2} I+W^{*} W\right)^{-1} T^{*} E(\{0\}) .
\end{aligned}
$$

We know $W T x=A W x=0$ for $x$ in $E(\{0\})$; therefore

$$
\begin{aligned}
\tau_{\mathbb{Q}_{H}}(Y)= & \tau_{\mathfrak{Q}_{H}}\left[(T E(\{0\}))^{*}, T E(\{0\})\right]+\tau_{\mathbb{Q}_{H}}\left(E(\{0\}) T E(\{0\}) T^{*} E(\{0\})\right) \\
& -E(\{0\}) T\left(\int_{0}^{\left\|W^{*} W\right\|} \frac{t^{2}}{t^{2}+\lambda^{2}} d E(\lambda)\right) T^{*} E(\{0\}),
\end{aligned}
$$

and

$$
\begin{gathered}
\tau_{\mathbb{Q}_{H}}\left\{\int_{[0]} 1 d E(\{0\}) T E(\lambda) T^{*} E(\{0\})-\int_{0^{+}}^{\left\|W^{*} W\right\|} \frac{t^{2}}{t^{2}+\lambda^{2}} d E(\{0\}) T E(\lambda) T^{*} E(\{0\})\right\} \\
=-\int_{0^{+}}^{\left\|W^{*} W\right\|} \frac{t^{2}}{t^{2}+\lambda^{2}} d \tau_{\mathbb{Q}_{H}}\left[E(\{0\}) T E(\lambda) T^{*} E(\{0\})\right] .
\end{gathered}
$$

As we have shown above, $\tau_{\mathfrak{Q}_{H}}\left[E(\{0\}) T E(\lambda) T^{*} E(\{0\})\right]$ is a finite measure. Since $t^{2} /\left(t^{2}+\lambda^{2}\right) \leqslant 1$, by the Lebesgue dominated convergence theorem this integral approaches 0 as $t$ goes to $0^{+}$. Thus as $t \rightarrow 0^{+}, \operatorname{tr}(Y) \rightarrow \operatorname{tr}\left[E(\{0\}) T^{*}, T E(\{0\})\right]$.

Our previous result was

(6) $\operatorname{tr}_{\mathfrak{Q}_{K}}\left(F(\sigma)\left[A^{*}, A\right] F(\sigma)\right)$

$$
=\lim _{t \rightarrow 0^{+}}\left\{(E(\sigma) \oplus F(\sigma))\left[\left.T \oplus A\right|_{J_{t}} ^{*},\left.(T \oplus A)\right|_{J_{t}}\right](E(\sigma) \oplus F(\sigma))\right\}
$$

for $\sigma \subset(0,\|W\|]$. In particular, if we divide the interval $(0,\|W\|]$ into half-open intervals of the form $\sigma_{n}=(\|W\| /(n+1),\|W\| / n]$, equation (6) is true for each $\sigma_{n}$.

Thus, we have

$$
\begin{aligned}
\operatorname{tr}\left[A^{*}, A\right]+\operatorname{tr}\left[E(\{0\}) T^{*}, T E(\{0\})\right] & \\
& =\sum \operatorname{tr}\left(F\left(\sigma_{n}\right)\left[A^{*}, A\right] F\left(\sigma_{n}\right)\right)+\operatorname{tr}\left[E(\{0\}) T^{*}, T E(\{0\})\right] .
\end{aligned}
$$

By Fatou's theorem this is bounded by

$$
\begin{gathered}
\underset{t \rightarrow 0^{+}}{\lim } \sum_{n} \operatorname{tr}\left\{\left(E\left(\sigma_{n}\right) \oplus F\left(\sigma_{n}\right)\right)\left[\left.(T \oplus A)\right|_{J_{t}} ^{*},\left.(T \oplus A)\right|_{J_{t}}\right]\left(E\left(\sigma_{n}\right) \oplus F\left(\sigma_{n}\right)\right)\right\} \\
+\operatorname{tr}\left[E(\{0\}) T^{*}, T E(\{0\})\right] .
\end{gathered}
$$


Using the property of normality of our trace, this expression becomes

$$
\begin{aligned}
& \underset{t \rightarrow 0^{+}}{\lim } \operatorname{tr} \sum_{n}\left\{\left(E\left(\sigma_{n}\right) \oplus F\left(\sigma_{n}\right)\right)\left[\left.(T \oplus A)\right|_{J_{t}}\right]\left(E\left(\sigma_{n}\right) \oplus F\left(\sigma_{n}\right)\right)\right\} \\
& +\underset{t \rightarrow 0^{+}}{\lim } \operatorname{tr}\left[E(\{0\}) t^{2}\left(t^{2} I+W^{*} W\right)^{-1} T^{*} T E(\{0\})\right. \\
& \left.-E(\{0\}) T t^{2}\left(t^{2} I+W^{*} W\right)^{-1} T^{*} E(\{0\})\right] \\
& =\lim _{t \rightarrow 0^{+}} \operatorname{tr}(E(0,\|W\|] \oplus F(0,\|W\|])\left[\left.(T \oplus A)\right|_{J_{t}} ^{*},\left.(T \oplus A)\right|_{J_{t}}\right] \\
& \times(E(0,\|W\|] \oplus F(0,\|W\|]) \\
& +\frac{\lim }{t+0^{+}} \operatorname{tr} E(\{0\})\left[E(\{0\}) t^{2}\left(t^{2} I+W^{*} W\right)^{-1} T^{*} T E(\{0\})\right. \\
& \left.-E(\{0\}) T t^{2}\left(t^{2} I+W^{*} W\right)^{-1} T^{*} E(\{0\})\right] E(\{0\}) \\
& \leqslant \frac{\lim }{t \rightarrow 0^{+}}\left\{\operatorname{tr}(E(0,\|W\|] \oplus F(0,\|W\|])\left[\left.(T \oplus A)\right|_{J_{t}} ^{*},\left.(T \oplus A)\right|_{J_{t}}\right]\right. \\
& \times(E(0,\|W\|] \oplus F(0,\|W\|])\} \\
& +\operatorname{tr}\left\{(E\{0\} \oplus 0)\left[J_{t}\left(T^{*} T \oplus A^{*} A\right) J_{t}-(T \oplus A) J_{t}\left(T^{*} \oplus A^{*}\right)\right](E\{0\} \oplus 0)\right\} \\
& =\lim _{t \rightarrow 0^{+}}\left\{\operatorname{tr}(E(0,\|W\|] \oplus F(0,\|W\|])\left[\left.(T \oplus A)\right|_{J_{t}} ^{*},\left.(T \oplus A)\right|_{J_{t}}\right]\right. \\
& \times(E(0,\|W\|] \oplus F(0,\|W\|]) \\
& \left.+\operatorname{tr}(E(\{0\}) \oplus 0)\left[\left.(T \oplus A)\right|_{J_{t}} ^{*},\left.(T \oplus A)\right|_{J_{t}}\right](E(\{0\}) \oplus 0)\right\} \\
& =\lim _{t \rightarrow 0^{+}}\left[\left.(T \oplus A)\right|_{J_{t}} ^{*},\left.(T \oplus A)\right|_{J_{t}}\right]=\operatorname{tr}\left[T^{*}, T\right] \text {. }
\end{aligned}
$$

2. In this section we prove the existence of an intertwining operator, $W$, which will enable us to transfer properties of an operator in one von Neumann algebra to an operator in another von Neumann algebra. To facilitate the proof of the intertwining theorem we use the following lemma.

LEMMA 2.1. Let $G$ be the finite union of multiconnected domains, each bounded by finitely many smooth Jordan curves. Let $A$ be an operator in $\mathbb{Q}_{K}$ whose spectrum lies in $G$, and let $F$ be a projection in $\mathcal{Q}_{K}$. For $z$ in $G \backslash \mathrm{sp}(A)$, let $W_{z}$ be the following operator in $B\left\{\left(Q^{2}\left(\chi_{G} \eta\right)\right) \otimes F, K\right\}: W_{z}(h \otimes x)=\left\langle h, \kappa_{z}\right\rangle(A-z I)^{-1} x$, where $\kappa_{z}$ is the Bergman kernel for $G$ [1]. Then $W_{z}^{*} Q_{K} W_{z^{\prime}}$ is contained in $B\left(Q^{2}\left(\chi_{G} \eta\right)\right) \otimes F Q_{K} F$ and $W_{z^{\prime}}\left(B\left(Q^{2}\left(\chi_{G} \eta\right)\right) \otimes F Q_{K} F\right) W_{z}^{*}$ is in $Q_{K}$.

Proof. It suffices to prove the lemma for the operator $Y_{z}$, where $Y_{z}(h \otimes x)=$ $\left\langle h, \kappa_{z}\right\rangle x$, since $(A-z I)^{-1}$ belongs to $\mathbb{Q}_{K}$. Let us call $\left\langle h, \kappa_{z}\right\rangle=L_{\kappa_{z}}(h)$. Thus $Y_{z}(h \otimes x)=L_{\kappa_{z}}(h) x$, and $Y_{z}^{*} x=\kappa_{z} \otimes x$. 
Let $C$ be an operator in $B\left(Q^{2}\left(\chi_{G} \eta\right)\right)$ and let $M$ belong to $Q_{K}$. We compute

$$
\begin{aligned}
& Y_{z^{\prime}}(C \otimes F M F) Y_{z}^{*}=\left\langle C \kappa_{z}, \kappa_{z^{\prime}}\right\rangle F M F \in \mathbb{Q}_{K}, \\
& Y_{z}^{*} M Y_{z^{\prime}}=\left(L_{z^{\prime}}, L_{z}^{*}\right) \otimes F M F \in B\left(Q^{2}\left(\chi_{G} \eta\right)\right) \otimes F Q_{K} F .
\end{aligned}
$$

INTERTWINING THEOREM. Let $G$ be a finite union of multiconnected domains each bounded by a finite union of smooth disjoint Jordan curves. Let $F$ be a finite projection in $Q_{K}$ and let $T=T_{z} \otimes F$ be contained in $B\left(Q^{2}\left(\chi_{G} \eta\right)\right) \otimes F Q_{K} F$. Let $A$ in $Q_{K}$ be such that $\operatorname{sp}(A)$ is contained in $G$ and

$$
\left\{q_{\alpha}(A) y: y \in F \text { and } q_{\alpha} \in Q\left(\operatorname{sp}\left(T_{z} \otimes F\right)\right)\right\}^{-}=K .
$$

We define $\operatorname{tr}_{Q \otimes F}(x \otimes M)=\operatorname{tr}(x) \operatorname{tr}_{\mathbb{Q}_{K}}(M)$ for $M \in F Q_{K} F$, and the first trace is the counting trace on $B\left(Q^{2}\right)$. Then there exists $a W$ in $B\left\{Q^{2}\left(\chi_{G} \eta\right) \otimes F, K\right\}$ such that:

(i) $W$ has dense range,

(ii) $W T=A W$,

(iii) $W W^{*}$ and $W^{*} W$ are in trace class.

(iv) $W\left\{B\left(Q^{2}\left(\chi_{G} \eta\right)\right) \otimes F Q_{K} F\right\} W^{*}$ is in $Q_{K}$ and $W^{*} Q_{K} W$ is in $B\left(Q^{2}\left(\chi_{G} \eta\right)\right) \otimes F Q_{K} F$.

Proof. For $u$ in $Q^{2}\left(\chi_{G} \eta\right) \otimes F$, define $\hat{u}: G \times F \rightarrow \mathbf{C}$ by $\hat{u}(z, x)=\left\langle u, \kappa_{z} \otimes x\right\rangle$, where $\kappa_{z}$ is the Bergman kernel of $G$. The function $\hat{u}$ is conjugate linear in $x$, linear in $u$, and, since $\kappa_{z}$ is conjugate analytic in $z, u$ is analytic in $z$.

Let $\Gamma$ be a finite union of smooth Jordan curves bounding $\operatorname{sp}(A)$ in $G$. The map $z \rightarrow \kappa_{z}$ is strongly continuous for $z$ on $\Gamma$. Thus

$$
|\hat{u}(z, x)| \leqslant\|u\|\left\|\kappa_{z} \otimes x\right\| \leqslant\|u\| M_{\Gamma}\|x\| \quad \text { where } M_{\Gamma}=\sup _{z \in \Gamma}\left\|\kappa_{z}\right\| .
$$

For fixed $z, \hat{u}(z, \cdot)$ is a bounded conjugate linear form on $F$ and is therefore given as an inner product. There exists a unique $h_{z}^{u}$ in $F$ such that $\hat{u}(z)=,\left\langle h_{z}^{u},\right\rangle$.

In this case

$$
\left\langle h_{z}^{u}, x\right\rangle=\left\langle u, \kappa_{z} \otimes x\right\rangle \text {. }
$$

The vector $h_{z}^{u}$, which is linear in $u$ by the uniqueness of the Riesz representation theorem, is analytic in $z$ since $u(z$,$) is. From (7) and (8) we see that \left\|h_{z}^{u}\right\| \leqslant\|u\| \cdot M_{\Gamma}$. For $u \in Q^{2}\left(\chi_{G} \eta\right) \otimes F$ of the form $f \otimes x, h_{z}^{u}=f(z) x$.

Define

$$
W u=-\frac{1}{2 \pi i} \int_{\Gamma}(A-z I)^{-1} h_{z}^{u} d z,
$$

where $\Gamma$ is a finite union of smooth Jordan curves bounding $\operatorname{sp}(A)$ in $G$. We have $W(f \otimes x)=-\frac{1}{2 \pi i} \int_{\Gamma}(A-z I)^{-1} h_{z}^{f \otimes x} d z=-\frac{1}{2 \pi i} \int_{\Gamma} f(z)(A-z I)^{-1} x d z=f(A) x$.

Thus, by (6'), $W$ has dense range. We will now show $W$ is bounded:

$$
\begin{aligned}
\|W u\|^{2} & =\left\|-\frac{1}{2 \pi i} \int_{\Gamma}(A-z I)^{-1} h_{z}^{u} d z\right\|^{2} \leqslant K_{\Gamma}\left\|(A-z I)^{-1}\right\| \cdot\left\|h_{z}^{u}\right\| \\
& \leqslant K_{\Gamma} \sup _{z \in \Gamma}\left\|(A-z I)^{-1}\right\|\|u\|\|\Gamma\|<+\infty,
\end{aligned}
$$

where $K_{\Gamma}=($ length of $\Gamma) / 2 \pi$. 
Note that for $f \otimes x$ in $Q^{2}\left(\chi_{G} \eta\right) \otimes F$,

$$
W\left(T_{z} \otimes F\right)(f \otimes x)=W\left(T_{z} f \otimes x\right)=W(z f \otimes x)=A F(A) x=A W(f \otimes x) .
$$

Thus $W\left(T_{z} \otimes F\right)=A W . W$ is the uniform limit of sums of the form $W_{z}$ where $W_{z}(h \otimes x)=\left\langle h, \kappa_{z}\right\rangle(A-z I)^{-1} x$. From Lemma 2.1 we see

$$
W\left[B\left(Q^{2}\left(\chi_{G} \eta\right)\right) \otimes F Q_{K} F\right] W^{*}
$$

is contained in $\mathbb{Q}_{K}$. Similar reasoning shows $W^{*} \mathbb{Q}_{K} W$ is in $B\left(Q^{2}\left(\chi_{G} \eta\right)\right) \otimes F Q_{K} F$. To show (iii), we compute the following:

$$
\begin{aligned}
\left\|W^{*} W\right\|_{\operatorname{tr}_{Q \otimes F}} & =\left\|\frac{1}{(2 \pi)^{2}} \iint W_{z}^{*} W_{z^{\prime}} d z d z^{\prime}\right\|_{\operatorname{tr}_{Q \otimes F}} \\
& \leqslant \frac{1}{(2 \pi)^{2}} \iint\left\|W_{z}^{*} W_{z^{\prime}}\right\|_{\operatorname{tr}} d|z| d\left|z^{\prime}\right| \\
& =\left.\frac{1}{(2 \pi)^{2}} \iint\left\|L_{z}^{*} L_{z^{\prime}} \otimes F(A-z I)^{-1 *}\left(A-z^{\prime} I\right)^{-1} F\right\|_{\operatorname{tr}_{Q \otimes F}} d|z| d\right|_{F} z^{\prime} \mid \\
& =\frac{1}{(2 \pi)^{2}} \iint\left|\left\langle\kappa_{z}, \kappa_{z^{\prime}}\right\rangle\right|\left\|F(A-z I)^{-1 *}\left(A-z^{\prime} I\right)^{-1} F\right\|_{\operatorname{tr}_{\mathbb{Q}_{K}}} d|z| d\left|z^{\prime}\right| \\
& \leqslant M \cdot \operatorname{tr}_{\mathbb{Q}_{K}}(F) .
\end{aligned}
$$

Also,

$$
\begin{aligned}
\left\|W W^{*}\right\|_{\operatorname{tr}_{\mathfrak{d}^{\mathfrak{d}}}} & =\left\|\frac{1}{(2 \pi)^{2}} \iint W_{z^{\prime}} W_{z}^{*} d z d z^{\prime}\right\|_{\operatorname{tr}_{\mathfrak{d}^{\prime} K}} \\
& \leqslant \frac{1}{(2 \pi)^{2}} \iint\left\|W_{z^{\prime}} W_{z}^{*}\right\|_{\operatorname{tr}_{\mathfrak{d}^{\prime} K}} d|z| d\left|z^{\prime}\right| \\
& =\frac{1}{(2 \pi)^{2}} \iint\left\|\left\langle\kappa_{z^{\prime}}, \kappa_{z}\right\rangle\left(A-z^{\prime} I\right)^{-1} F(A-z I)^{-1 *}\right\| \operatorname{tr}_{\mathbb{Q}_{K}} d|z| d\left|z^{\prime}\right| \\
& \leqslant M \cdot \operatorname{tr}_{\mathfrak{Q}_{K}}(F) .
\end{aligned}
$$

3. Let $T$ belong to $\mathscr{Q}_{H}$ and let $E$ be a finite projection in $\mathscr{Q}_{H}$. We will denote the space spanned by $\{p(T) E: p \in P\}$ by $V$. Clearly $V$ is invariant with respect to $T$. Note that $\left.V Q_{H}\right|_{V}$ is again a von Neumann algebra. Let $\tau^{\prime}$ be the restriction of $\tau_{\mathbb{Q}_{H}}$ to $\left.V Q_{H}\right|_{V}$

LEMMA 3.1. Let $T$ be an invertible operator in $\mathbb{Q}_{H}$, and let $E$ be a finite projection in $\mathcal{Q}_{H}$. Then the orthogonal projection whose range is $R\left(T E T^{-1}\right)$ is finite with trace equal to the trace of $E$.

Proof. Let $Q$ be the orthogonal projection whose range is $\overline{R\left(T E T^{-1}\right)}$. We claim there is an isometry in $\mathcal{Q}_{H}$ from $E$ onto $\overline{R\left(T E T^{-1}\right)}$. Once this is established, the finiteness of $E$ implies $Q$ is finite, and $\operatorname{tr}(Q)=\operatorname{tr}(E)$. 
Consider the polar decomposition of the operator $T E$ in $\mathcal{Q}_{H} . T E=V \sqrt{E T^{*} T E}$, where $U$ is in $Q_{H} \cdot U$ is an isometry from $\overline{R\left((T E)^{*}\right)}$ onto $\overline{R(T E)} . \overline{R(T E)}$ $=\overline{R\left(T E T^{-1}\right)}$ and $\overline{R\left((T E)^{*}\right)}=E$. Therefore, $Q=U E U^{*}$ implies $Q$ is finite. Indeed, $E=U^{*} Q U$, and so $\operatorname{tr}(E)=\operatorname{tr}(Q)$.

Corollary 3.2. Let $T$ be invertible. The projection $P_{k}$, whose range is $\overline{R\left(T^{K} E\right)}$, is finite for every $K$.

Recall that $V$ is the closure of the space $\{p(T) E: p \in P\}$.

LEMMA 3.3. The operator $V$ belongs to $\mathbb{Q}_{H}$ and is the strong limit of an increasing sequence of finite projections in $\mathbb{Q}_{H}$.

Proof. Let $E_{0}$ be the space $E$, and let $E_{n}$ be the space spanned by $E$, $T[E], \ldots, T^{n}[E]$ for each $n$. Since $E$ and $T$ belong to $\mathcal{Q}_{H}, E_{n}$ is a finite projection in $\mathcal{Q}_{H}$ for every $n$. The $E_{n}$ increase strongly to $V$ and the conclusion follows.

LEMMA 3.4. Let $T$ be hyponormal and assume $\tau_{\mathfrak{Q}_{H}}\left[T^{*}, T\right]<+n$. Then $T=T_{0} \oplus T_{n}$ where $H=H_{0} \oplus H_{n} . T_{0}=\left.T\right|_{H_{0}}$ is a completely nonnormal operator and $T_{n}=\left.T\right|_{H_{n}}$ is a normal operator. Furthermore, $H_{0}$ and $H_{n}$ lie in $Q_{H}$. If $\sigma$ is a Borel subset of $R$ which is bounded away from 0 , and $E(\sigma)$ is the corresponding spectral projection for $\left[T_{0}^{*}, T_{0}\right]$, then $E(\sigma)$ is a finite projection in $\mathbb{Q}_{H}$.

LEMMA 3.5. Let $V$ be an invariant space for $T$, a hyponormal operator, where $T$ and $V$ belong to $\mathbb{Q}_{H}$. Let $H=V_{0} \oplus V_{n}$ where $V_{0}$ is the subspace of $H$ upon which $T V$ is completely nonnormal and $V_{n}$ is the subspace where $T V$ is normal. Then $T$ is normal on $V_{n}$ also.

Proof. Clearly $V_{n}$ is contained in $V$. For $u$ in $V_{n}$ we have $\left\|V T^{*} u\right\|=\|T V u\|=$ $\|T u\| \geqslant\left\|T^{*} u\right\|$ since $T$ is hyponormal. Since $V$ is a projection, $\left\|T^{*} u\right\| \geqslant\left\|V T^{*} u\right\|$. For $u$ in $V_{n}, V T^{*} u=T^{*} u$. Since $V_{n}$ is contained in $V, T$ and its adjoint agree with $T V$ and its adjoint, respectively. Thus $T$ is normal on $V_{n}$.

The Subspace Dominance Theorem. Let the hyponormal operator $T$ be effectually rationally cyclic with respect to $V$. Then $\operatorname{tr}\left[T^{*}, T\right] \leqslant \operatorname{tr}\left[\left(\left.T\right|_{V}\right)^{*},\left(\left.T\right|_{V}\right)\right]$.

Proof. We may assume that $\operatorname{tr}\left[\left(\left.T\right|_{V}\right)^{*},\left(\left.T\right|_{V}\right)\right]$ is finite. Since $T$ is effectually rationally cyclic with respect to the invariant space $V,\left\{x: p \in P^{\prime}, p(T) x \in V\right\}^{-}=H$. Polynomials with rational coefficients are dense in the set of all polynomials so there is a countable subset $P^{\prime \prime}$ of $P^{\prime}$ such that $\left\{x: p \in P^{\prime \prime}, p(T) x \in V\right\}^{-}=H$. For $p \in P^{\prime \prime}$, define $V_{p}=\{x \in H: p(T) x \in V\}$. Let $V_{1}=V$. Clearly $V_{p}$ is invariant for $T$ and there are a countable number of $V_{p}$. Set $s_{0}=1, s_{1}=p_{1}, s_{2}=p_{1} p_{2}, s_{3}=p_{1} p_{2} p_{3}$, etc. We have $V=V_{s_{0}}$ and $V_{s_{i}}=\left\{x \in H: s_{i}(T) x \in V\right\}$. Trivially $V_{s_{i}}$ contains $V_{p_{i}}$ for every $i$. The $V_{s_{i}}$ are nested and increase strongly to the identity. Each polynomial $s_{i}$ contains $s_{i-1}$ as a factor. By choosing $P^{\prime \prime}$ suitably, we may assume that $s_{n} / s_{n-1}$ is a monic linear polynomial. Thus $V=V$ and $V_{n+1}=\left\{x:\left(T-a_{n+1}\right) x \in V_{n}\right\}$, where $a_{n+1} \in \mathbf{C}$. We will prove the following chain of inequalities.

$$
\operatorname{tr}\left[T^{*}, T\right] \leqslant \frac{\lim }{n}\left(\operatorname{tr}\left[\left(T V_{n}\right)^{*},\left(T V_{n}\right)\right] \leqslant \operatorname{tr}\left[\left(\left.T\right|_{V}\right)^{*},\left(\left.T\right|_{V}\right)\right]\right)
$$


Since $V_{n}=\left\{x:(I-V) p(T) x=0\right.$ for some $\left.p \in P^{\prime}\right\}, V_{n}$ is the kernel of an operator in $\mathbb{Q}_{H}$ and so belongs to $\mathbb{Q}_{H}$.

In proving the first inequality in (9), we assume $\operatorname{tr}\left[\left(T V_{n}\right)^{*},\left(T V_{n}\right)\right]$ is frequently finite, otherwise the result follows trivially. By Lemma 3.4 there exists a sequence of finite orthogonal projections whose partial sum increases to the identity on the space on which $T V_{n}$ is completely nonnormal. Let $\left(V_{n}\right)_{0}$ be the space upon which $T \mid V_{n}$ is completely nonnormal. It suffices to calculate the trace of the self-commutators of the operators restricted to $\left(V_{n}\right)_{0}$. Since the $V_{n}$ are increasing up to $I_{H}$, without loss of generality we may assume the sum of the sequence of finite orthogonal projections is increasing up to $I_{H}$.

Let $\left\{E_{i}\right\}$ be a sequence of finite orthogonal projections such that $\sum_{i=1}^{N} E_{i}$ is increasing up to $I$, the identity. Since $\tau$ is a normal trace, we find

$$
\operatorname{tr}\left[T^{*}, T\right]=\sum_{i=1}^{\infty} \operatorname{tr}\left(E_{i}\left[T^{*}, T\right] E_{i}\right)
$$

and

$$
\operatorname{tr}\left[\left(T V_{n}\right)^{*},\left(T V_{n}\right)\right]=\sum_{i=1}^{\infty} \operatorname{tr}\left(E_{i}\left[\left(T V_{n}\right)^{*},\left(T V_{n}\right)\right] E_{i}\right)
$$

Next it will be shown that

$$
\lim _{n} \operatorname{tr}\left(E_{i}\left(T V_{n}\right)\left(T V_{n}\right)^{*} E_{i}\right)=\operatorname{tr}\left(E_{i} T T^{*} E_{i}\right)
$$

and

$$
\lim _{n} \operatorname{tr}\left(E_{i}\left(T V_{n}\right) *\left(T V_{n}\right) E_{i}\right)=\operatorname{tr}\left(E_{i} T^{*} T E_{i}\right)
$$

These equalities will imply

$$
\lim _{n} \operatorname{tr}\left(E_{i}\left[\left(T V_{n}\right)^{*},\left(T V_{n}\right)\right] E_{i}\right)=\operatorname{tr} E_{i}\left[T^{*}, T\right] E_{i} .
$$

We have

$$
E_{i}\left(T V_{n}\right)\left(T V_{n}\right)^{*} E_{i}=E_{i} T V_{n} T^{*} E_{i} \text { and }\left\langle E_{i} T V_{n} T^{*} E_{i} x, x\right\rangle=\left\|V_{n} T^{*} E_{i} x\right\| .
$$

Setting $Y=T^{*} E_{i} x$, we find $\left\|V_{n} T^{*} E_{i} x\right\|=\left\|V_{n} y\right\|$. The $\left\{V_{n}\right\}$ are increasing up to $H$ and therefore $\left\{E_{i} T V_{n} T^{*} E_{i}\right\}$ are increasing up to $E_{i} T T^{*} E_{i}$. Since $\tau$ is normal,

$$
\lim _{n} \tau\left(E_{i} T V_{n} T^{*} E_{i}\right)=\tau\left(E_{i} T T^{*} E_{i}\right) \text {. }
$$

We will now show $\lim _{n} \tau\left(E_{i}\left(T V_{n}\right)^{*}\left(T V_{n}\right) E_{i}\right)=\tau\left(E_{i} T^{*} T E_{i}\right)$. Since

$$
\begin{aligned}
\left\{\operatorname{tr}\left(E_{i}\left(T V_{n}\right)^{*}\left(T V_{n}\right) E_{i}\right)\right\} & =\left\{\operatorname{tr}\left(E_{i} V_{n} T^{*} T V_{n} E_{i}\right)\right\} \\
& =\operatorname{tr}\left\{\left(V_{n} E_{i} V_{n} T^{*} T\right)\right\}=\operatorname{tr}\left(T V_{n} E_{i} V_{n} T^{*}\right)
\end{aligned}
$$

and $\operatorname{tr}\left(E_{i} T^{*} T E_{i}\right)=\operatorname{tr}\left(T E_{i} T^{*}\right)$, it suffices to prove $\lim _{n} \operatorname{tr}\left(T V_{n} E_{i} V_{n} T^{*}\right)=\operatorname{tr}\left(T E_{i} T^{*}\right)$. Consider the following set of equivalent relationships:

$$
\begin{aligned}
& \lim _{n} \operatorname{tr}\left(T V_{n} E_{i} V_{n} T^{*}\right)=\operatorname{tr}\left(T E_{i} T^{*}\right), \\
& \lim _{n} \operatorname{tr}\left(T V_{n} E_{i} V_{n} T^{*}\right)-\operatorname{tr}\left(T E_{i} T^{*}\right)=0,
\end{aligned}
$$


(12) $\operatorname{tr}\left[T\left(E_{i}-V_{n} E_{i} V_{n}\right) T^{*}\right]$ is approaching 0 ,

(13) $\operatorname{tr}\left(T\left[\left(I-V_{n}\right) E_{n} T^{*}\right]\right)+\operatorname{tr}\left(T V_{n}\right)\left[E_{i}\left(I-V_{n}\right) T^{*}\right]$ is approaching 0 .

It therefore suffices to prove (13) true.

Clearly

$$
\operatorname{tr}\left(T V_{n}\left(E_{i}\left(I-V_{n}\right) T^{*}\right)\right)=\operatorname{tr}\left[\left(T^{*} T V_{n} E_{i}\right)\left(E_{i}\left(I-V_{n}\right)\right)\right] .
$$

If $A$ and $B$ belong to a von Neumann algebra with a trace defined on it, $|\operatorname{tr}(A B)|^{2} \leqslant\left|\operatorname{tr} A A^{*}\right| \cdot\left|\operatorname{tr} B^{*} B\right|$; therefore,

$$
\left|\operatorname{tr}\left(T^{*} T V_{n} E_{i}\right)\left(E_{i}\left(I-V_{n}\right)\right)\right|^{2} \leqslant \operatorname{tr}\left(T^{*} T V_{n} E_{i} V_{n} T^{*} T\right) \cdot \operatorname{tr}\left[\left(I-V_{n}\right) E_{i}\left(I-V_{n}\right)\right] .
$$

Since $E_{i}$ belongs to trace class and $T^{*}, T$ and $V$ are all bounded operators, $T^{*} T V_{n} E_{i} V_{n} T^{*} T$ belongs to trace class. We claim $\lim _{n} \operatorname{tr}\left(E_{i}\left(I-V_{n}\right) E_{i}\right)$ goes to 0 . Since the $V_{n}$ are increasing up to $I$ and $\tau$ is normal,

$$
\begin{aligned}
\left\{\lim _{n} \operatorname{tr}\left[E_{i}\left(I-V_{n}\right) E_{i}\right]\right\} & =\left\{\operatorname{tr}\left(E_{i} I E_{i}\right)-\lim _{n} \operatorname{tr}\left(E_{i} V_{n} E_{i}\right)\right\} \\
& =\left\{\operatorname{tr}\left(I E_{i}\right)-\lim _{n} \operatorname{tr}\left(V_{n} E_{i}\right)\right\}=0 .
\end{aligned}
$$

The value

$$
\tau\left(T\left(I-V_{n}\right) E_{i} T^{*}\right)=\operatorname{tr}\left(T^{*} T\left(I-V_{n}\right) E_{i}\right)=\operatorname{tr}\left(E_{i} T^{*} T\left(I-V_{n}\right) E_{i}\right)
$$

and

$$
\left|\operatorname{tr}\left(E_{i} T^{*} T\left(I-V_{n}\right) E_{i}\right)\right|^{2} \leqslant \operatorname{tr}\left(T^{*} T E_{i} T^{*} T\right) \cdot \operatorname{tr}\left(E_{i}\left(I-V_{n}\right) E_{i}\right) .
$$

Using an argument similar to the previous one, $\operatorname{tr}\left(T^{*} T E_{i} T^{*} T\right)<+\infty$ and $\operatorname{tr}\left[E_{i}\left(I-V_{n}\right) E_{i}\right]$ approaches 0 . Therefore,

$$
\lim _{n} \operatorname{tr}\left(E_{i}\left[\left(T V_{n}\right)^{*},\left(T V_{n}\right)\right] E_{i}\right)=\operatorname{tr}\left(E_{i}\left[T^{*}, T\right] E_{i}\right) .
$$

Using this we see

$$
\begin{aligned}
\operatorname{tr}\left[T^{*}, T\right] & =\operatorname{tr} \sum_{i=1}^{\infty} E_{i}\left[T^{*}, T\right] E_{i}=\sum_{i=1}^{\infty} \operatorname{tr}\left(E_{i}\left[T^{*}, T\right] E_{i}\right) \\
& =\sum_{i=1}^{\infty} \lim _{n} \operatorname{tr}\left(E_{i}\left[\left(T V_{n}\right)^{*},\left(T V_{n}\right)\right] E_{i}\right) \leqslant \frac{\lim }{n} \sum_{i=1}^{\infty} \operatorname{tr}\left(E_{i}\left[\left(T V_{n}\right)^{*},\left(T V_{n}\right)\right] E_{i}\right) \\
& =\frac{\lim }{n} \operatorname{tr}\left[\left(T V_{n}\right)^{*}, T V_{n}\right]
\end{aligned}
$$

The inequality is a consequence of Fatou's theorem.

In order to complete the proof of this theorem we need to establish

$$
\frac{\lim }{n} \operatorname{tr}\left[\left(T V_{n}\right)^{*},\left(T V_{n}\right)\right] \leqslant \operatorname{tr}\left[\left(\left.T\right|_{V}\right)^{*},\left(\left.T\right|_{V}\right)\right]
$$

Let $B_{n+1}=\left.T\right|_{V_{n+1}}$, where $V_{n+1}=\left\{x:\left(T-a_{n+1} I\right) x \in V_{n}\right\}$. Clearly $B_{n+1}$ is effectually rationally cyclic with respect to $V_{n}$. It suffices to show

$$
\operatorname{tr}\left[B_{n+1}^{*}, B_{n+1}\right] \leqslant \operatorname{tr}\left[B_{n}^{*}, B_{n}\right] \text { for arbitrary } n .
$$


Equivalently, it is enough to show that if $K$ is an invariant space for $T$, a hyponormal operator in $\mathcal{Q}_{H}$, and $\{x: T x \in K\}^{-}=H$, then $\operatorname{tr}\left[T^{*}, T\right] \leqslant \operatorname{tr}\left[(T K)^{*}, T K\right]$.

Without loss of generality we may assume $N(T)=0$. To see this, let $K^{\prime}=\{x+n$ : $x \in K, n \in N(T)\}$. The space $N$ is a reducing space for $T$ since $T$ is hyponormal. Since $T K=T K^{\prime}$ and $(T K)=\left(T K^{\prime}\right)^{*},\left[(T K)^{*},(T K)\right]=\left[\left(T K^{\prime}\right)^{*},\left(T K^{\prime}\right)\right]$. Furthermore, $T H$ contained in $K$ implies $T H$ is in $K^{\prime}$. The space $K^{\prime}$ is invariant for $T$ since both $N$ and $K$ are invariant. It suffices, therefore, to prove $\operatorname{tr}\left[T^{*}, T\right] \leqslant$ $\operatorname{tr}\left[\left(T K^{\prime}\right)^{*},\left(T K^{\prime}\right)\right]$. Further, we may assume $N(T)$ is in $K$. Thus, $N$ is a reducing space for $\left.T\right|_{K^{\prime}}$. Since both $T$ and $\left.T\right|_{K^{\prime}}$ are normal on $N$, the foregoing does not affect the trace of either commutator.

By Lemma 3.4 there exist $\left\{E_{n}\right\}$, finite projections increasing up to $K$. Let $F_{n}=\{x$ : $\left.T x \in E_{n}\right\}$. Our claim is that $F_{n}$ is a finite projection for every $n$. Consider $B_{n}=\left.T\right|_{F_{n}}$. Since $N(T)=0, N\left(B_{n}\right)$ must equal 0 . The operator $\left.T F_{n}\right|_{F_{n}}$ has no nullity and $R\left(T F_{n}\right)$ is contained in $E_{n}$. Thus $T F_{n}=E_{n} T F_{n}=S_{n} R_{n}$ where $R_{n}$ is a positive hermitian operator and $S_{n}$ is a partial isometry. Since $T$ has no nullity, $N\left(R_{n}\right)=I-$ $F_{n}$. Also, $R\left(S_{n}\right)$ is contained in $R\left(E_{n}\right)$ and $\overline{R\left(S_{n}^{*}\right)}=N\left(S_{n}\right)=\overline{R\left(R_{n}\right)}$. We conclude that $F_{n}=\overline{R\left(R_{n}\right)}=\overline{R\left(S_{n}\right)}$. Since $\overline{R\left(S_{n}^{*}\right)}=S_{n}^{*} S_{n}, S_{n} S_{n}^{*}=\overline{R\left(S_{n}\right)}$ and $S_{n} S_{n}^{*}$ is contained in $E_{n}, F_{n}$ is equivalent to a subspace of $E_{n}$. For each $n, E_{n}$ is finite implies $F_{n}$ is finite. The $F_{n}$ are increasing up to the identity on $H$.

Let $U_{n}=\left\{x+y: x \in K, y \in F_{n}\right\} . T H$ is contained in $K$ implying $U_{n}$ is invariant for $T$. Clearly $K$ is in $U_{n}$ and $U_{n}-K$ is in $F_{n}$. Thus $U_{n}-K$ is finite and $\operatorname{tr}\left[\left(\left.T\right|_{U_{n}}\right)^{*},\left(\left.T\right|_{U_{n}}\right)\right]=\operatorname{tr}\left[\left(\left.T\right|_{K}\right)\right]$. The $U_{n}$ are increasing up to the identity. By an argument similar to the one used to prove the first inequality in (9), we have

$$
\operatorname{tr}\left[\left(\left.T\right|_{K}\right)^{*},\left(\left.T\right|_{K}\right)\right]=\frac{\lim }{n} \operatorname{tr}\left[\left(\left.T\right|_{U_{n}}\right)^{*},\left(\left.T\right|_{U_{n}}\right)\right] \leqslant \operatorname{tr}\left(T^{*}, T\right]
$$

Combining the two inequalities we have $\operatorname{tr}\left[T^{*}, T\right] \leqslant \operatorname{tr}\left[\left(\left.T\right|_{V}\right)^{*},\left(\left.T\right|_{V}\right)\right]$.

4. In this final section we prove our major result. Utilizing the results of the preceding sections we prove that the trace of the self-commutator of a hyponormal operator is bounded above by a multiplicity factor times the area of the spectrum of the operator. Formally, we have

THEOREM 4.1. Let $A$ be a hyponormal operator in $\mathcal{Q}_{H}$ with effectually rational multiplicity s. Then $\left[A^{*}, A\right]$ is in trace class and $\operatorname{tr}\left[A^{*}, A\right] \leqslant(s / \pi) \operatorname{area}(\operatorname{sp}(A))$. If $f$ is a function analytic on $\operatorname{sp}(A)$ and if $f(A)$ is hyponormal, then

$$
\operatorname{tr}\left[f(A)^{*}, f(A)\right] \leqslant \frac{s}{\pi} \int_{\operatorname{sp}(A)}\left|f^{\prime}\right|^{2} d \eta .
$$

Proof. Let $U$ be a bounded open set in $\mathrm{C}$ such that $\operatorname{sp}(A) \subset U, \eta(U)-\eta(\operatorname{sp}(A))$ is small and $U$ is bounded by a finite number of smooth Jordan curves. Let $H^{\prime}$ be the space spanned by $\left\{\sum_{i=1}^{n} q_{i}(A) e_{i}: e_{i} \in E\right.$ and $\left.q_{i} \in Q(U)\right\}$. Clearly $H^{\prime}$ is in $\mathbb{Q}_{H}$. Let $B=\left.A\right|_{H^{\prime}}$. From [16] we see that $B$ has its spectrum contained in $U . B$ is hyponormal and rationally cyclic with respect to $E$ on $H^{\prime}$. By the subspace dominance theorem, $\left[\operatorname{tr} A^{*}, A\right] \leqslant \operatorname{tr}\left[B^{*}, B\right]$. 
Let $T=T_{z} \otimes E$ be the operator acting on the Hilbert space $K=Q^{2}\left(\chi_{U} \eta\right) \otimes E$. From the results in the $I_{\infty}$ case [7], we know

$$
\begin{aligned}
\operatorname{tr}\left[T^{*}, T\right] \operatorname{tr}\left[T_{z}^{*} \otimes E, T_{z} \otimes E\right] & =\operatorname{tr}\left[T_{z}^{*} T_{z} \otimes E-T_{z} T_{z}^{*} \otimes E\right] \\
& =\operatorname{tr}\left[T_{z}^{*}, T_{z}\right] \cdot \operatorname{tr}(E)=\left(\kappa_{E} / \pi\right) \eta(U),
\end{aligned}
$$

where $\operatorname{tr}(E)=\kappa_{E}$.

To complete the proof we need to exhibit $W$ in $B\left(K, H^{\prime}\right)$, an intertwiner between $T$ and $B$ that satisfies the following hypotheses of the intertwining theorem $Z$ :

(i) $W^{*} W$ and $W W^{*}$ are trace class operators.

(ii) $W$ has dense range.

(iii) $W T=B W$.

(iv) $V Q_{H^{\prime}} V^{*}$ is in $Q_{K}$ and $V^{*} Q_{K} V$ is contained in $Q_{H^{\prime}}$ where $W=V \sqrt{W^{*} W}$.

(v) $\tau_{\mathfrak{Q}_{H}}\left(V X V^{*}\right)=\tau_{\mathbb{Q}_{K}}(X)$ for all $X$ in trace class in $\mathbb{Q}_{K}$.

The map $W: Q^{2}\left(\chi_{U}, \eta\right) \times E \rightarrow H^{\prime}$ defined by $W\left(f \otimes e_{\alpha}\right)=f(A) e_{\alpha}$ satisfies properties (i)-(v). The range of $W$ is dense in $H^{\prime}$ since $B$ is rationally cyclic with respect to $E$ on $H$. Thus

$$
\operatorname{tr}\left[A^{*}, A\right] \leqslant\left(\kappa_{E} / \pi\right) \eta(U) .
$$

If we let $E$ vary over all the finite projections in $\mathbb{Q}_{H}$ with respect to which $A$ is effectually rationally cyclic, we find, upon taking the infimum of both sides of (15), that $\operatorname{tr}\left[A^{*}, A\right] \leqslant(s / \pi) \eta(U)$. Let $\eta(U)$ approach $\eta(\operatorname{sp}(A))$ and the first result is proved.

As for the second statement in the theorem, since any $f$ analytic on $J$ can be uniformly approximated by rationals, $W[f(T)]=f(B) W$; therefore, we have an intertwining map, and coupled with the $I_{\infty}$ result that $\operatorname{tr}\left[f(T)^{*}, f(T)\right]=\frac{1}{\pi} \int\left|f^{\prime}\right|^{2} d \eta$ [1], the proof is essentially the same as the above.

\section{REFERENCES}

1. N. Aronszajn, Theory of repreducing kernels, Trans. Amer. Math. Soc. 68 (1950), 337-404.

2. C. A. Berger and B. I. Shaw, Self-commutators of multicyclic operators are always trace class, Bull. Amer. Math. Soc. 79 (1973), 1193-1199.

3. Manfred Breuer, Fredholm theories in von Neumann algebras. I, Math. Ann. 178 (1968), 243-254.

4. Jacques Dixmier, Les $C^{*}$-algèbres et leurs représentations, Gauthier-Villars, Paris, 1964.

5. R. G. Douglas, Banach algebras techniques in operator theory, Academic Press, New York, 1972.

6. P. R. Halmos, A Hilbert space problem book, Van Nostrand, New York, 1967.

7. __ Introduction to Hilbert space and the theory of spectral multiplicity, 2nd ed., Chelsea, New York, 1957.

8. B. I. Shaw, Trace class self-commutators of hyponormal operators, Doctoral Dissertation, Yeshiva University, New York, 1973.

Department of Mathematics, Yeshiva University, New York, New York 10033

Department of Mathematics, Mercy College, Dobbs Ferry, New York 10522 